\title{
Nuevos stents con drogas. ¿Solución a Barcelona 2006?
}

Un pequeño paso para un hombre, un gran salto para la humanidad

Neil Amstrong, 20 de Julio, 1969

\section{Dr. Milton Alcaíno}

Recibido el 24 de agosto de 2009, aceptado el 24 de septiembre de 2009

Rev Chil Cardiol 2009; 28: 389-391

Desde los inicios de la angioplastía coronaria hasta la actualidad, se ha producido un considerable avance en el tratamiento percutáneo de la enfermedad coronaria. La angioplastía con balón, limitada en sus comienzos a un número relativamente reducido de pacientes, se asoció, entre otros problemas, a restenosis $^{1-2}$. El uso de los stents, redujo en forma importante esta complicación, al disminuir la remodelación negativa del vaso, la cual se produce después de la angioplastía con balón ${ }^{3}$. Sin embargo, la restenosis siguió siendo un problema, especialmente debido a proliferación neointimal ${ }^{4}$. La restenosis secundaria a los stents sin drogas, varía entre un $15 \%$ a un $60 \%$, dependiendo del cuadro clínico, la presencia de diabetes, insuficiencia renal o características de la lesión: ubicación, vasos pequeños o longitud de la lesión ${ }^{5}$, y obliga, en muchas ocasiones, a nuevos procedimientos de revascularización.

Los stents con drogas (SD), fueron introducidos a comienzos de esta década y generaron al poco tiempo una gran expectativa ${ }^{6}$, pues disminuyeron entre un $60 \%$ a $70 \%$ las revascularizaciones secundarias a restenosis post-stent ${ }^{7-8}$. Debido a estos resultados, los SD comenzaron a utilizarse amplia-mente, tanto en pacientes con características

similares a los de los estudios randomizados, efectuados hasta ese momento ("on label"), como en pacientes de otras características, en general de mayor riesgo ("off label") ${ }^{9}$. Al poco tiempo, comenzaron a publicarse reportes respecto a trombosis de los $\mathrm{SD}^{10}$. En el Congreso Europeo de Cardiología del año 2006 en Barcelona, Camenzind reportó un meta-análisis de los estudios realizados con SD, tanto con sirolimus como con paclitaxel, encontrando un exceso de muerte o infarto del miocardio de un $38 \%$ para los stents con sirolimus y un $16 \%$ para los stents con paclitaxel, comparados con stents corrientes ${ }^{11}$, atribuyéndose fundamentalmente como causa de estos eventos, a la trombosis de estos dispositivos. Nordmann, por otra parte, sugirió un aumento de mortalidad de causa no cardíaca para los stents con sirolimus a los 2 y 3 años de seguimiento $^{12}$. Aunque estos estudios no fueron bien realizados, crearon una gran preocupación en cuanto a la seguridad y eficacia de los SD. Como conse cuencia, hubo nuevos análisis, con mejor información de los estudios realizados hasta el momento, los que mostraron seguridad y eficacia de los SD, aunque existía un pequeño porcentaje de trombosis muy tardía, que no oscurecía los efectos benéficos de estos stents ${ }^{13}$. Se utilizaron definiciones respecto a trombosis de stent de acuerdo al consenso ARC (Academic Research Consortium) ${ }^{14}$. Se publicaron registros de la experiencia en el "mundo real" con SD los que también revelaron un buen perfil de seguridad $^{15}$. Se recomendó el uso de clopidogrel por un año, para los pacientes en que se implantaran SD y hubo un mayor estímulo para el desarrollo de nuevos stents ${ }^{16-17}$.

La trombosis de los SD es multifactorial ${ }^{18}$. Depende de las características del paciente: diabetes, disfunción ventricular, síndrome coronario agudo, resistencia a clopidogrel y/o aspirina; características de la lesión; factores relacionados al procedimiento:

Correspondencia: Dr. Milton Alcaíno

Hospital DIPRECA.

Correo Eléctronico:miltonalc@hotmail.com 
aposición adecuada del stent, numero de stents, disección; duración del tratamiento con clopidogrel y finalmente las características del stent. El polímero de los SD tendría un importante rol en este fenómeno, ya que puede producir un proceso inflamatorio, que retarda la endotelización y favorece la trombosiscorrientes ${ }^{19}$, por lo mismo, están en desarrollo nuevos SD con polímeros de diferentes características o sin polímero.

Recientemente, se ha comenzado a utilizar un nuevo stent de Medtronic, el Endeavor Resolute. Este stent, posee un nuevo polímero (BioLinx), el cual tiene un componente hidrofílico, para mayor biocompatibilidad y liberación rápida de la droga, además de un componente hidrofóbico para producir una mejor dispersión del medicamentocorrientes ${ }^{20}$.

En la presente edición Caorsi y colaboradores, presentan un interesante estudio observacional con el stent Resolute y aunque el estudio es pequeño, de un sólo centro, no existiendo comparación con otro tipo de stent, especialmente con drogas, los resultados tienen un bajo número de eventos adversos y ausencia de trombosiscorrientes ${ }^{21}$. Sería importante tener información a mayor plazo, con un mayor número de pacientes e idealmente comparados con otro SD. Hay un estudio pequeño con este stent, cuyos resultados a 24 meses revelan un bajo porcentaje de restenosis y sin fenómenos de trombosiscorrientes ${ }^{22}$, estando actualmente en desarrollo estudios con un mayor numero de pacientes. Otros stents han mostrado buenos resultados respecto a restenosis, aunque no han eliminado el problema de la trombosiscorrientes ${ }^{16}$.

Estudios de SD sin polímero, aunque pequeños, han mostrado también resultados similares a los stents con polímero en cuanto a trombosicorrientes ${ }^{23}$. Respecto a los stents con polímeros biodegradables, uno de los estudios con un tipo de estos stents (Nobori) comparado con stent Taxus, en un reducido número de pacientes es prometedorcorrientes ${ }^{24}$, aunque otro estudio de SD con polímero biodegradable (LEADERS), con un mayor número de pacientes, comparado con stent Cypher, no mostró grandes diferencias a 9 mesescorrientes ${ }^{17}$. Finalmente el uso de stents degradablescorrientes ${ }^{25}$ pudiera ser la solución a este problema, pero se requiere mayor información y especialmente a largo plazo.

Por lo tanto, Barcelona, conocido por Gaudi, por su equipo de futbol y por los paseos en La Rambla y alrededores, después del año 2006, se hizo conocido por el impacto que significaron los resultados presentados por los Drs. Camenzind y Nordmann, además de los fuertes comentarios del Dr. Yusuf 26. Lo sucedido en ese Congreso produjo una amplia revisión de los trabajos realizados hasta ese momento con $\mathrm{SD}$, se comenzó a utilizar la definición ARC, respecto a la trombosis de los stents: aguda, precoz, tardía y muy tardía; definitiva, probable y posible, quedando en claro también, la seguridad de estos stents ${ }^{27}$ estimulándose además la investigación con nuevos stents, es decir, hubo un "gran salto". Actualmente, se sabe que la trombosis y especialmente la muy tardía, no sólo existe con los SD, sino también puede ocurrir con los stents sin drogas ${ }^{28}$ y en el caso de los SD, la trombosis muy tardía es probablemente un fenómeno asociado a inflamación, en la que el polímero jugaría un importante rol. Por lo tanto, aunque aun se requiere mayor investigación, existen respuestas al "incidente" ocurrido en Barcelona.

\section{Referencias}

1. HOLMES DR JR, HOLUBKOV R, VLIETSTRA RE, KELSEY SF, REEDER GS, DORROS $G$, et al. Comparison of complications during percutaneous transluminal coronary angioplasty from 1977 to 1981 and from 1985 to 1986: the National Heart, Lung, and Blood Institute Percutaneous Transluminal Coronary Angioplasty Registry. J Am Coll Cardiol 1988; 12: 1149-1155.

2. DETRE K, HOLUBKOW R, KESLEY S, BOURASSA M, WILLIAMS D, HOLMES D JR, et al. One-year follow-up results of the 1985 - 1986 National Heart, Lung, and Blood Institute's Percutaneous Transluminal Coronary Angioplasty Registry. Circulation 1989; 80: 421-428.

3. MINTZ GS, POPMA JJ, PICHARD AD, KENT KM, SATLER LF, WONG $C$, et al. Arterial remodeling after coronary angioplasty. A serial intravascular ultrasound study. Circulation 1996; 94: 35-43

4. HOFFMANN R, MINTZ GS, DUSSAILLANT GR, POPMA JJ, PICHARD AD, SATLER LF, et al. Patterns and mechanism 
of in-stent restenosis. A serial intravascular ultrasound study. Circulation 1996; 94: 1247-1254.

5. CUTLIP DE, CHAUHAN MS, BAIM DS, HO KK, POPMA JJ, CARROZZA JP, et al. Clinical restenosis after coronary stenting: perspectives from multicenter clinical trials. J Am Coll Cardiol 2002; 40: 2082-2089.

6. SOUSA JE, COSTA MA, ABIZAID A, ABIZAID AS, FERES F, PINTO IM, et al. Lack of neointimal proliferation after sirolimus-coated stent in human coronary arteries: a quantitave coronary angiography and three-dimensal intravascular ultrasound study. Circulation 2001; 103: 192-195.

7. MOSES JW, LEON MB, POPMA JJ, FITZGERALD PJ, HOLMES DR, O'SHAUGHNESSY C, et al. Sirolimus-eluting stents versus standard stents in patients with stenosis in a native coronary artery. N Engl J Med 2003; 349: 1315-1323.

8. STONE GW, ELLIS SG, COX DA, HERMILLER J, O'SHAUGHNESSY C, MANN JT, et al. A polymer-based, paclitaxel-eluting stent in patients with coronary artery disease. N Engl J Med 2004; 350: 221- 231.

9. ONG AT, SERRUYS PW, AOKI J, HOYE A, VAN MIEGHEM CA, RODRIGUEZ-GRANILLO GA, et al. The unrestricted use of paclitaxel- versus sirolimus-eluting stents for coronary artery disease in an unselected population: one-year results of the Taxus-Stent Evaluated at Rotterdam Cardiology Hospital (T-SEARCH) registry. J Am Coll Cardiol. 2005; 45: 1135-1141.

10. MCFADDEN EP, STABILE E, REGAR E, CHENEAU E, ONG AT, KINNAIRD T, et al. Late thrombosis in drug-eluting coronary stents after discontinuation of antiplatelet therapy. Lancet 2004; 364: 1519-1521.

11. CAMENZIND E. STEG PG, WIJNS W. Safety of drug-eluting stents: A meta-analysis of first generation DES programs. European Society of Cardiology Annual Scientific Conference, 2006.

12. NORDMANN AJ, BRIEL M, BUCHER HC. Mortality in randomized controlled trial comparing drug-eluting vs bare-metal stents in coronary artery disease. A meta-analisys. Eur Heart J 2006; 27: 2784-2814.

13. STONE GW, MOSES JW, ELLIS SG, SCHOFER J, DAWKINS KD, MORICE MC, et al. Safety and efficacy of sirolimus and paclitaxel-eluting coronary stents. N Engl J Med 2007; 356: 998-1008.

14. CUTLIP DE, WINDECKER S, MEHRAN R, BOAM A, COHEN DJ, VAN ES GA, et al. Clinical end points in coronary stents trials. A case for standardized definitions. Circulation 2007; 115: 2344-2351.

15. MAURI L, SILBAUGH TS, WOLF RE, ZELEVINSKY K, LOVETT $A$, ZHOU Z, et al. Long-term clinical outcomes after drug-eluting and bare-metal stenting in Massachusetts. Circulation 2008; 118: 1817-1827.

16. STONE GW, MIDEI M, NEWMAN W, SANZ M, HERMILLER JB, WILLIAMS J, et al Comparison of an everolimus- eluting stent in patients with coronary artery disease: a randomized trial. JAMA 2008; 299: 1903-1913.

17. WINDECKER S, SERRUYS PW, WANDEL $S$, BUSZMAN P, TRZNADEL S, LINKE A, et al. Biolimus-eluting stent with biodegradable polymer versus sirolimus-eluting stent with durable polymer for coronary revascularisation(LEADERS): a randomized non-inferiority trial. Lancet 2008; 372: 1163-1173.

18. WINDECKER S, MEIER B. Late coronary stent thrombosis, Circulation 2007; 116: 1952-1965.

19. VIRMANI R, GUAGLIUMI G, FARB A, MUSUMECI G, GRIECO N, MOTTA T, et al. Localized hypersensitivity and late coronary thrombosis secondary to a sirolimus-eluting stent. Should we be cautious? Circulation 2004; 109: 701-705.

20. UDIPI K, MELDER R, CHEN M, CHENG P, HEZI-YAMIT A, SULLIVAN $C$, et al. The next generation Endeavor Resolute Stent: role of the Biolinx Polymer System. Eurointerv 2007; 3: 137-139.

21. CAORSI C, BARRA V, KAUFFMANN R, PINEDA F. Nueva generación de stents, con polímero bio-compatiple: resultados y seguimiento clínico.

Rev Chil Cardiol 2009; 28: 357-362

22. SERRYS PW. Resolute's place in current clinical practice. Why is Resolute different from Endeavor. europcronline. 20.05.2009.

http://www.europcronline.com/fo/lecture/view_slide.php? idCongress $=5 \&$ id $=7637$.

23. MEHILLI J, BYRNE RA, WIECZOREK A, IIJIMA R, SCHULZ S, BRUSKINA $O$, et al. Randomized trial of three rapamycin-eluting stents with different coating strategies for the reduction of coronary restenosis. Eur Heart J 2008; 29: 1975-1982.

24. BARBATO E. Nobori 1: long term results: two years phase $1 \& 2$ and three years phase1.euroPCRonline.20.05.2009. http://www.europcronline.com/fo/lecture/view_slide.php? id Congres $=5 \&$ id $=7102$.

25. ORMISTON JA, SERRUYS PW, REGAR E, DUDEK D, THUESEN L, WEBSTER MW, et al. A bioasorbable everolimus-eluting coronary stent system for patients with single de-novo coronary lesions (ABSORB): A retrospective open-label trial. Lancet 2008; 371: 899-907.

26. Studies linking drug-eluting stents to increase mortality /MI spark impassioned pleas for reason and calls for calm. http://www.theheart.org/article/736863.do.

27. KIRTANE AJ, GUPTA A, IYENGAR S, MOSES JW, LEON MB, APPLEGATE R, et al. Safety and efficacy of drug-eluting and bare metal stents: comprehensive meta-analysis of randomized trial and observational study. Circulation 2009; 119: 3198-3206.

28. RAMOS AR, MORICE MC, LEFEVRE T. Late and very late stent thrombosis can also occur with bare metal stents. Catheter Cardiovasc Interv 2007; 70: 229-232. 